\title{
An algorithm for surface segmentation of aircraft structural parts
}

\author{
Jinglin Xu, Guolei Zheng \\ Sch. of Mech. Eng. \& Automation \\ Beihang University \\ Beijing, China \\ e-mail: jinglin.xu@163.com, \\ zhengguolei@buaa.edu.cn
}

\author{
Baorui Du \\ Technology center \\ Shenyang Aircraft Industry (Group) \\ Corporation Ltd. \\ Shenyang, China \\ e-mail: sacdbr@sohu.com
}

\author{
Faming $\mathrm{Wu}$ \\ R\&D center, EMD department \\ Linde(China)Forklift truck Co. Ltd. \\ Xiamen, China \\ e-mail: wufaming580@126.com
}

\begin{abstract}
The paper presents an algorithm that aims to extract machining features from aircraft structural parts' 3D model based on both face- and workability-based approaches under certain processing coordinate system. The proposed algorithm consists of the following 4 major steps for surface region segmentation: extracting model's face sets and $Z$ axis vector, pre-processing the face sets, analyzing the sampling and constructing the patches. Meanwhile, principles of the algorithm are presented accordingly. The verified feasibility study of the developed method is also presented.
\end{abstract}

Keywords- surface segmentation; feature recognition; aircraft structural parts;

\section{INTRODUCTION}

The trends of integrated, large-scale, and high accuracy aircraft parts booms the development of computer aided numerical control (CNC) machining technology. As a matter of fact, aircraft structural parts accounted for about $75 \%$ aircraft CNC machining parts. Thus makes the complex surface machining becoming a hot topic in the field of computer aided manufacturing. Surface segmentation will be quite useful in manufacturing where complicated surfaces are used.

Researchers worldwide have dedicated to developing a variety of methods for surface segmentation. Pierre P. Lefebvre and Bert Lauwers ${ }^{[1]}$, described a subdivision procedure for a STL model on behalf of an automated multiaxis milling operations planning system. Sunil VB and Pande $\mathrm{SS}^{[2]}$, presented a new approach for region segmentation on discrete meshes(STL type) based on the computation of Gauss $(\mathrm{K})$ and $\operatorname{Mean}(\mathrm{H})$ curvature measures. Wang Dazhen $^{[3]}$, introduced a cylindrical milling method of divided area. Lin Jie-qiong ${ }^{[4]}$, put forward a new algorithm of freeform surface subdivision planning by means of the combination of the curvature based method and the fuzzy Cmeans method, which split free-form surface into surface pieces so as to promote the polishing efficiency.

Currently, there are three essential ways associated with aircraft structural parts' machining: 3 -axis, $3 \& 2$ axis, and 5$\operatorname{axis}^{[5-6]}$. No matter which axis is assigned, the most fundamental problem stems from the lack of appropriate surface segmentation methods. Limitation of the approaches proposed above leads to extra requirements for optimizing the segmentation of surface before geometrical feature identification.

\section{PURPOSE}

The research reported in this paper is motivated by the above observation. The work focuses on developing methods for automatic segmentation of various aircraft structural parts' surfaces. The rest of the paper is organized as follows: Section III describes theoretical foundation of surface segmentation. Section IV presents the methodology and rules for surface segmentation. Various case studies and analysis are presented in Section V. Conclusions from this research work are summarized in Section VI.

\section{PRINCIPLES}

\section{A. Basic theory}

Points on the aircraft structural parts' surfaces have various attributes like position, curvature, normal vector, etc. Suppose $a_{i}(i=1,2, \ldots, n)$ to be the attributes of the point $p_{f}\left(a_{1}, a_{2} \ldots, a_{i}, \cdots a_{n}\right)$ and classify the attributes into three different categories as below:

1) Intrinsic attribute: If there is an attribute that belongs to any point on the surface $\left(\forall p_{f}, \exists a \in p_{f}\right)$, then this attribute is defined as the intrinsic attribute of $p_{f}$. For example, every point owns a "position" intrinsic attribute.

2) Associated attribute: This kind of attribute depends on the surface where it attached, such as "normal vector", "tangent vector", "curvature" (including Gaussian curvature, mean curvature and principal curvatures), "radius of curvature" and so on. The associated attributes won't be meaningful or have its geometrical significance unless the surface exists.

3) External attribute/Domain attribute: This sort of attribute is indicated when necessary, like "gray degree", "color" etc.

These three attributes listed above are independent from each other; under no circumstances will they replace each other. The face point $p_{f}\left(a_{1}, a_{2} \ldots, a_{i}, \cdots a_{n}\right)$ consists of different attribute values. If point $p_{f}$ has only one associated attribute value, and no other possible associated attribute values exist, then $p_{f}$ is defined as an inner point of 
its relative surface. Nevertheless, if $p_{f}$ has more than one value related to the associated attribute, then $p_{f}$ is defined as a boundary point of the surface.

Whether a point is workable with a certain cutter during a machining process is judged by creating radial line that starts from $p_{f}$ and then intersecting it with the faces excluding its own related face. If the radial line and model has no intersection point that lies on other faces, $p_{f}$ is workable, otherwise, $p_{f}$ is un-workable.

\section{B. Model surface segmentation process}

Automatic numerical control machining depends much on the result of model feature recognition. The segmenting procedure can be done by separating surfaces in accordance with different engineering semantics, i.e. whether a face is workable during certain machining process. Surface segmentation process within a 3D model is shown in Fig.1, from which we can see that by collecting all the surfaces that belonged to one model into surfaces set, analyzing the surface type and then using the methods presented below can the patch models be returned.

The following instance of T-type slot processing aims to illustrate the purpose of segmentation. As shown in Fig.2, under a assigned machining coordinate, mainly two procedures are involved. First, use the cylindrical milling cutter illustrated in Fig.2(a) for handling rectangular slot, after which the segmentation of bottom face in the rectangular slot are accomplished. As shown in Fig.4, patches $f_{1}, f_{5}$ and $f_{9}$ are acquired during above process. Second, transfer to the T-type cutter in Fig.2 (b) to finish Ttype slot, after which patches $f_{2}, f_{3}, f_{4}, f_{6}, f_{7}$ and $f_{8}$ are generated, as shown in Fig.3.

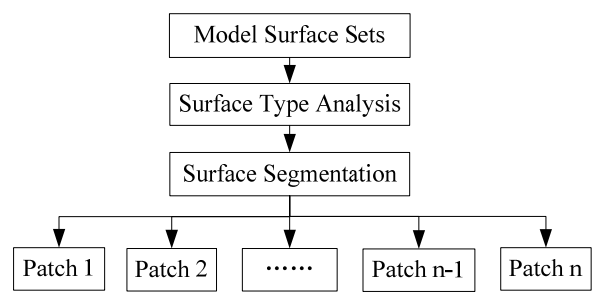

Figure 1. Model Surface Segmentation Process.

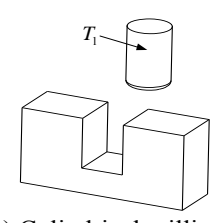

(a) Cylindrical milling cutter

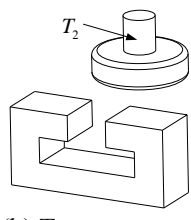

(b) T-type cutter
Figure 2. T-type slot machining process

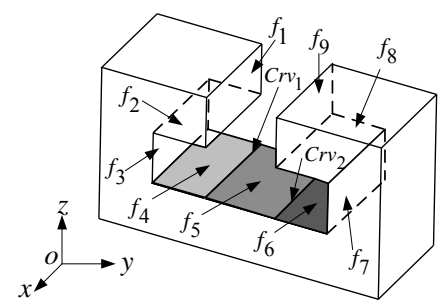

Figure 3. T-type slot segmenting process

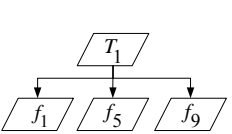

(a)

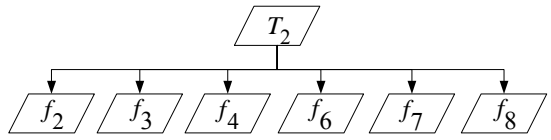

(b)
Figure 4. Cutter-Patch relevance.

Thus, the relation between a certain cutter and patches are conspicuous. Fig.4 shows the relevance between the cutter and the patches during the processes described above. Obviously, the cylindrical milling cutter $\left(T_{1}\right)$ can machine patches $f_{1}, f_{5}$ and $f_{9}$ accordingly, and T-type cutter $\left(T_{2}\right)$ can cut the others consequently. This patch-based expression of machining features can not only describe the relations in an accurate way, but also present it more comprehensively. In addition, extra information such as manufacturing precision, processing datum face, etc. can be added when necessary. As a result, the processed feature can be expressed as follows:

$$
\begin{aligned}
& F=\left(f_{1} \cup f_{2} \cup \ldots \cup f_{k}\right) \cup\left(T_{1} \cup T_{2} \cup \ldots \cup T_{1}\right) \\
& \cup\left(T f_{1,1} \cup T f_{2,1} \cup \ldots \cup T f_{l, k}\right) \cup\left(A_{1} \cup A_{2} \cup \ldots \cup A_{m}\right) \\
& \cup\left(C_{1} \cup C_{2} \cup \ldots \cup C_{n}\right)
\end{aligned}
$$

Where, $f_{i}$ is the patch obtained from surface segmentation, $T_{i}$ is the relative cutter, $T f_{i j}$ indicates the relevance between cutter $T_{i}$ and patch $f_{i}, \mathrm{~A}_{i}$ and $C_{i}$ expresses the adjacent relationship between different patches.

\section{ALGORITHM}

The algorithm for aircraft structural surface segmentation is of great importance because of its significant influence on the efficiency and reliability of the entire feature recognition process. According to the principles addressed in section III, the algorithm is designed as follows and the flowchart shown in Fig.5 is taken as a detailed depiction of how surface segmentation in this paper works.

Step 1: Extract the model face sets and $Z$ axis vector. This step is implemented by selecting the $3 \mathrm{D}$ model and machining coordinate interactively, and then the model face sets and $\mathrm{Z}$ axis vector are achieved consequently.

Step 2: Pre-Process the model face sets. In this step, the key point is how to evaluate whether a facet is workable or not. According to the definition of workability mentioned above, if a face does not satisfied the definition, it should be 
rejected out of the face sets to be handled. Otherwise, it will be added into face sets to be segmented.

Step 3: Analyze the sampling. The parametric domain in both $u$ and $v$ directions will be obtained during this process. A method based on the parameter domain grid sampling will be applied here to get the sampling points. The visibility of the sampling points is judging by creating radial lines starting from the sampling points and then intersecting it with the faces excluding the face it relies on. When the point of intersection exists, the sampling point is defined as invisible. The algorithm only focuses on the visible points.

Step 4: Construct the patches. The construct process is listed below:

1) Detect the boundary points: The detection of the boundary points includes area both inside the face and right on the edge. The boundary point sets must be the union of the sets inside the face and the sets on the edge.

2) Interpolate the boundary points: After acquiring the boundary points that belong to the same boundary of the patch, the interpolation process should be conducted. It is guaranteed by sequencial boundary points analysis and careful parameterization of the faces.

3) Construct the patches: The patch is surrounded by the boundary, so the construction of the patches is equivalent to using the boundary to limit the face so as to extract patches that are suitable for a certain cutter to finish the machining process. Finally, the patch model is returned and the calculation result is exported as well.

At present, this method is designed for aircraft structural parts' face segmentation under the 3 -axis machining mode, which can be used to reconstruct feature during the processing procedures. When 5 -axis machining mode is taken into consideration, just by changing the $\mathrm{Z}$ vector can this paper's method be realized.

\section{IMPLEMENTATION}

The proposed approach has been implemented in a prototype system, developed under Windows XP. The 3D

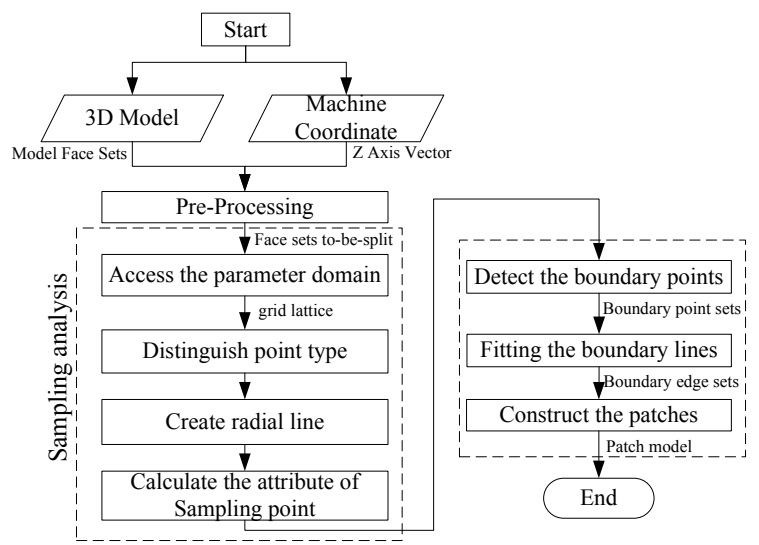

Figure 5. Flowchart of aircraft structure face segmentation display platform is CATIA V5 R19, which provides program-developing tool CAA. The developing language is Visual C++ 6.0. Two aircraft structural part models, featuring with invagination and multi-subsidence slot in Fig. 6.and Fig. 7 separately, are utilized to verify the capability of the method proposed in this paper. The statistical verification is shown in Table I. Fig.7 shows boundary points of the invagination's bottom face. Invagination segmentation results are shown in Fig.8, and Fig.9 shows the result of multi- subsidence slot feature segmentation.

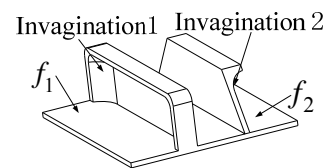

Figure 6. Invagination feature.

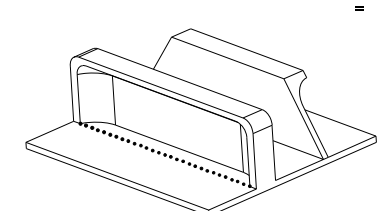

(a)
Figure 7. Invagination feature.

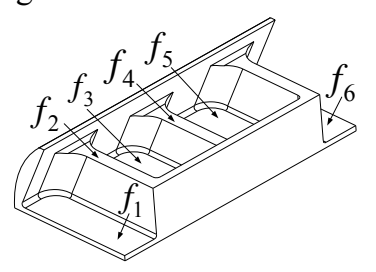

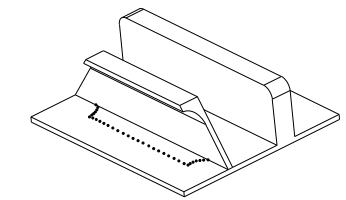

(b)
Figure 8. Result of extracting boundary points .

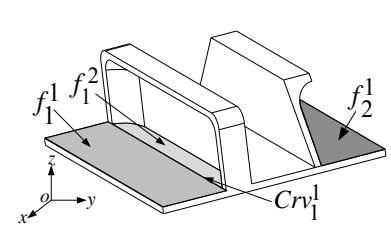

(a)

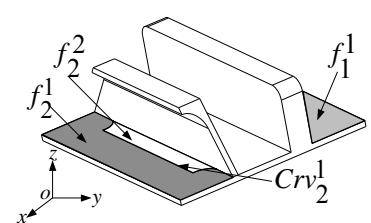

(b)
Figure 9. Invagination Segmentation.

TABLE I. SEGMENTATION RESULT

\begin{tabular}{|c|c|c|c|c|}
\hline \multirow{2}{*}{$\begin{array}{l}\text { Testing } \\
\text { Model }\end{array}$} & \multirow{2}{*}{ Face } & \multicolumn{3}{|c|}{ Segmentation Result } \\
\hline & & Boundary & Type & Patch \\
\hline \multirow{2}{*}{ Fig.9 } & $f_{1}$ & Crv 1 & Line & $f_{1}^{1}, f_{1}^{2}$ \\
\hline & $f_{2}$ & Crv $\frac{1}{2}$ & Curve & $f \frac{1}{2}, f_{2}^{2}$ \\
\hline \multirow{6}{*}{ Fig.10 } & $f_{1}$ & $\operatorname{Crv}{ }_{1}^{1}$ & Line & $f_{1}^{1}, f_{1}^{2}$ \\
\hline & $f_{2}$ & $\operatorname{Crv} \frac{1}{2}, \operatorname{Crv} \frac{2}{2}$ & Line & $f_{2}^{1}, f_{2}^{2}, f_{2}^{3}$ \\
\hline & $f_{3}$ & $\operatorname{Crv}_{3} \frac{1}{3}$ & Line & $f_{3}^{1}, f_{3}^{2}$ \\
\hline & $f_{4}$ & Crv $\frac{1}{4}$ & Line & $f_{4}^{1}, f_{4}^{2}$ \\
\hline & $f_{5}$ & Crv $\frac{1}{5}$ & Line & $f_{5}^{1}, f_{5}^{2}$ \\
\hline & $f_{6}$ & $\operatorname{Crv} \frac{1}{6}$ & Line & $f_{6}^{1}, f_{6}^{2}$ \\
\hline
\end{tabular}




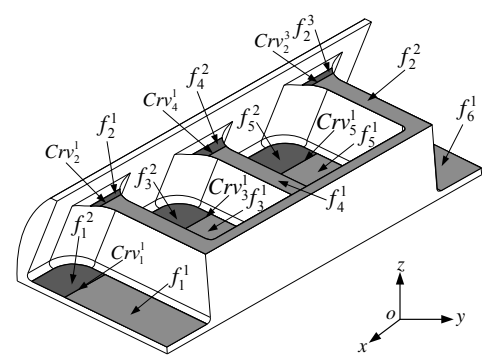

Figure 10. Multi- subsidence slot feature segmentation.

As shown in Fig.8, there are 29 boundary points that were got from the bottom face $\left(f_{1}\right)$ of invagination 1 , and the distance between an actual boundary point and the theoretical boundary line is calculated to analyze the error statistical result, as shown in Fig.11. Both boundary points' error $\left(E_{\text {boundary }}\right)$ and internal boundary points' error $\left(E_{\text {internal }}\right)$ are calculated. Like-wise, Fig. 12 shows the result of 41 boundary points' error verification results. Error statistics include maximum error $E_{\max }$, minimum error $E_{\min }$, error range $\Delta \mathrm{E}$ and average error $E_{\text {avg. }}$. Results are shown in Table III. As the boundaries of multi- subsidence slot feature segmentation are all straight lines, therefore the results will not go into details here.

The accuracy of the boundary point is set as $0.001 \mathrm{~mm}$. From Table II and Table III, $E_{\text {boundary }}=0<1 \mathrm{e}-3$, $E_{\text {internal }}=5.697 \mathrm{e}-4<1 \mathrm{e}-3$ and $E_{\max }=8.578 \mathrm{e}-4<1 \mathrm{e}-3$, which shows that the method is qualified to meet engineering requirements. When the boundary points are adequate enough, the interpolated boundary line can fulfill the segmentation accuracy.

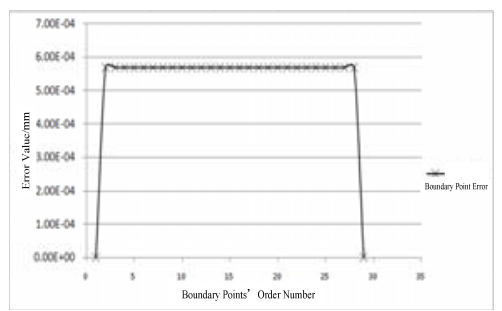

Figure 11. $f_{1}$ - Boundary Points Error.

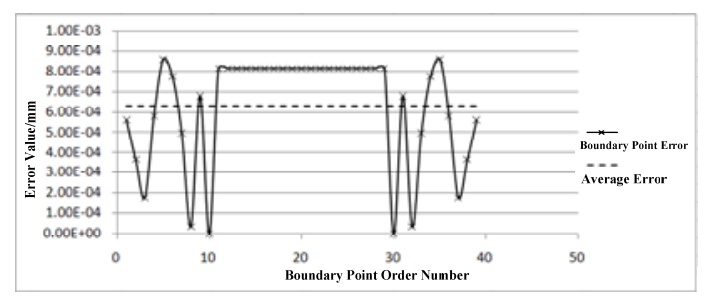

Figure 12. $f_{2}$ - Boundary Points Error.
TABLE II. $\quad f_{1}$-BOUNDARY POINTS ERROR ANALYSIS

\begin{tabular}{cc}
\hline $\boldsymbol{E}_{\text {boundary }} / \mathbf{m m}$ & $\boldsymbol{E}_{\text {internal }} / \mathbf{m m}$ \\
\hline 0 & $5.697 \mathrm{e}-4$ \\
\hline
\end{tabular}

TABLE III. $\quad f_{2}$-BOUNDARY POINTS ERROR ANALYSIS

\begin{tabular}{cccc}
\hline $\boldsymbol{E}_{\mathbf{m a x}} / \mathbf{m m}$ & $\boldsymbol{E}_{\mathbf{m i n}} / \mathbf{m m}$ & $\Delta \boldsymbol{E} / \mathbf{m m}$ & $\boldsymbol{E}_{\text {avg }} / \mathbf{m m}$ \\
\hline $8.578 \mathrm{e}-4$ & 0 & $8.578 \mathrm{e}-4$ & $6.287 \mathrm{e}-4$ \\
\hline
\end{tabular}

\section{CONCLUSION}

In this research a new approach to segment the aircraft structural parts' surfaces under 3-axis machining mode has been proposed. Considering the workability of the surface, the reported algorithm is capable of splitting the surface into several patches, which prepares for feature recognition and automatic NC programming. The method has been utilized by Shenyang Aircraft Industry (Group) Co. Ltd., achieving outstanding outcomes. However, this method only deals with boundary with curvature continuity, and lacks of the ability to process polygonal line without curvature continuity. Moreover, it can only handle one self-closing boundary. Further research will focus on: (1) solving the questions left and applying the methodology to feature recognition; (2) improving robustness of the algorithm proposed in this paper.

\section{ACKNOWLEDGMENT}

This work was supported by the National Science and Technology Major Project (NSTMP, No. 2012ZX04010051).

\section{REFERENCES}

[1] PP. Lefebvre and B. Lauwers, "STL model segmentation for multiaxis machining operations planning,". Computer-Aided Design \& Applications, vol. 1, 2004, pp.277-284.

[2] Sunil. VB, Pande. SS, "Automatic recognition of features from freeform surface CAD models,". Computer-Aided Design, vol. 40, 2008, pp.502-517.

[3] Wang Da-zhen, "Introduction on NC Machining of Integer impeller,". Mechanical Engineer, 2003, pp.34-36.

[4] Lin Jie-qiong and Wang Yi-qiang,"Subdivision planning of complex free-form surface,’Journal of Jilin University,vol.37,2007,pp.386-390.

[5] Yu Fangfang, "Research and Development of Rapid NC Machining Programming System for Aircraft Integral Panel,". School of Mechanical Engineering \& Automation, Beihang University, Beijing, China, 2009.

[6] Yu Fangfang, ZhengGulei, RenWenjie, et al, "Techniques of machining feature mapping from design-by-feature model,". Journal of Beijing University of Aeronautics and Astronautics, vol.33, 2007, pp.842-845. 